\title{
An unusual inflammatory lesion of the jejunum in a patient with late onset immunoglobulin deficiency
}

\author{
S. E. Williams \\ M.B. B.S., M.R.C.P. \\ Department of Medicine, Hope Hospital (University of Manchester School of Medicine), \\ Salford M6 8HD, Lancs
}

\begin{abstract}
Summary
A 40-year-old male patient is reported in whom a grossly inflamed thickened $70-\mathrm{cm}$ segment of jejunum complicated a 'late onset' immunoglobulin deficiency state. The pathogenesis is unknown, but extensive occlusion of the vascular supply to the lesion may have contributed to its development.
\end{abstract}

\section{Introduction}

Late onset immunoglobulin deficiency states are associated with a variety of gastrointestinal manifestations including steatorrhoea, bacterial overgrowth in the small bowel, giardiasis and nodular lymphoid hyperplasia. Although inflammatory disease of the intestine in this condition has been recorded, associated thromboses of mesenteric arteries and veins has not. Such a patient is reported below.

\section{Case history}

In 1967 a Caucasian male accountant, aged 40 years, developed watery diarrhoea, fever, malaise and painful mouth ulcers. After extensive investigation the only abnormality found was the presence of Giardia lamblia in the stools, and the patient improved dramatically on treatment with metronidazole. In 1968 he had an uncomplicated cholecystectomy for gall stones. From 1968 to 1972 he had further episodes of giardiasis, some associated with severe mouth ulceration, diarrhoea, weight loss, night sweats and latterly episodes of colicky left hypochondrial pain. These attacks were treated moderately successfully with courses of mepacrin or prednisolone.

A further attack in 1975 was accompanied by severe left hypochondrial pain and a thickened loop of jejunum became palpable for the first time. A laparotomy revealed a $70-\mathrm{cm}$ segment of thickened, oedematous and congested jejunum with thickened mesentery and many soft enlarged lymph nodes, but no resection was performed. Subsequently he remained reasonably well until February 1977 when he was admitted to Hope Hospital, Salford, with recurrent symptoms of subacute intestinal obstruction. He was underweight with mouth ulcera- $\frac{3}{\circ}$. tion and a tender loop of jejunum was still palpable of in the left hypochondrium.

\section{Investigations}

Most of the investigations carried out since 1971 had been normal, but the ESR remained persistently elevated between 30 and $50 \mathrm{~mm}$ in one hr. A barium $\vec{T}$ meal in 1972 showed a constant narrowing in the $\mathbb{P}$ second part of the duodenum with thickened mucosal folds and small nodular filling defects in many areas $\stackrel{\varrho}{2}$ of the small intestine (Fig. 1). Duodenoscopy at the time revealed a pale, coarsely granular duoden

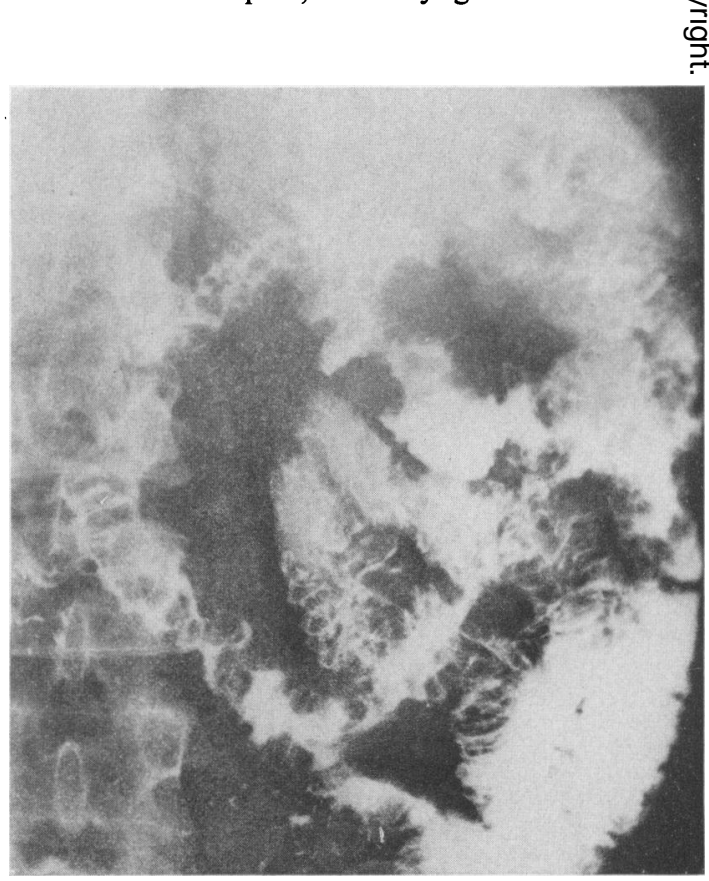

Fig. 1. Barium meal showing numerous small nodular filling defects in many areas of the small intestine, characteristic of nodular lymphoid hyperplasia. 
mucosa. Jejunal biopsy showed stunted villi, and in view of this he was given a gluten-free diet but failed to improve. He was found to have marked deficiency of all serum immunoglobulins in 1972 and the concentrations of these have remained consistently low with values ranging between zero and $0.56 \mathrm{~g} / 1$ for IgA (normal range $0.9-4.5 \mathrm{~g} / \mathrm{l}$ ), 0.1 and $0.5 \mathrm{~g} / \mathrm{l}$ for IgM (normal range $0.6-2.8 \mathrm{~g} / \mathrm{l}$ ), and 3.8 and $8.0 \mathrm{~g} / \mathrm{l}$ for IgG (normal range 8-18 $\mathrm{g} / \mathrm{l}$ ). In addition, salivary and jejunal aspirate immunoglobulin concentrations were also extremely low (IgA 0-0.44 g/l). In 1976 a repeat jejunal biopsy revealed the features of nodular lymphoid hyperplasia with large lymphoid follicles within the lamina propria (Fig. 2).

\section{Progress and recent treatment}

Following admission to Hope Hospital, he was treated with an elemental diet to 'rest' the bowel and a further course of metronidazole therapy. Despite this and $12 \mathrm{u}$. of fresh frozen plasma given over a 2-month period, there was no clinical improvement and only transient changes in his serum immunoglobulin concentrations. During this time he developed an acute seronegative arthritis affecting both knees and the left ankle which settled on indomethacin therapy. He had developed a similar transient arthropathy in 1973.
Because of continuing subacute obstruction a laparotomy was performed in May 1977. Marked adhesions were found with thickened jejunum extending from $20 \mathrm{~cm}$ beyond the duodeno-jejunal flexure to mid-jejunum. There were also numerous enlarged mesenteric lymph nodes. The remaining bowel appeared normal and the diseased jejunum was resected.

Macroscopically the lesion resembled Crohn's disease (Fig. 3) with thickened mucosal folds, nodular areas and fissuring. Histologically the bowel wall was considerably thickened owing to transmural chronic inflammation, oedema and fibrosis, but no tuberculoid granulomata were identified in the wall or mesenteric nodes. There were extensive undermined flat ulcers lined with granulation tissue. Surprisingly the mesenteric arteries and veins were both extensively occluded by old organized thrombus (Figs 4, 5). There was no evidence of arteritis, the appearances being those of diffuse chronic ischaemia due to mesenteric occlusion without obvious cause.

Following the resection he improved dramatically, gaining weight and losing all his symptoms. His ESR fell to normal and he has remained well since operation without further treatment, although his immunoglobulin levels have not significantly increased.

FIG. 2. Jejunal biopsy. A large lymphoid follicle is shown within the lamina propria with normal overlying epithelium. This is characteristic of nodular lymphoid hyperplasia. 


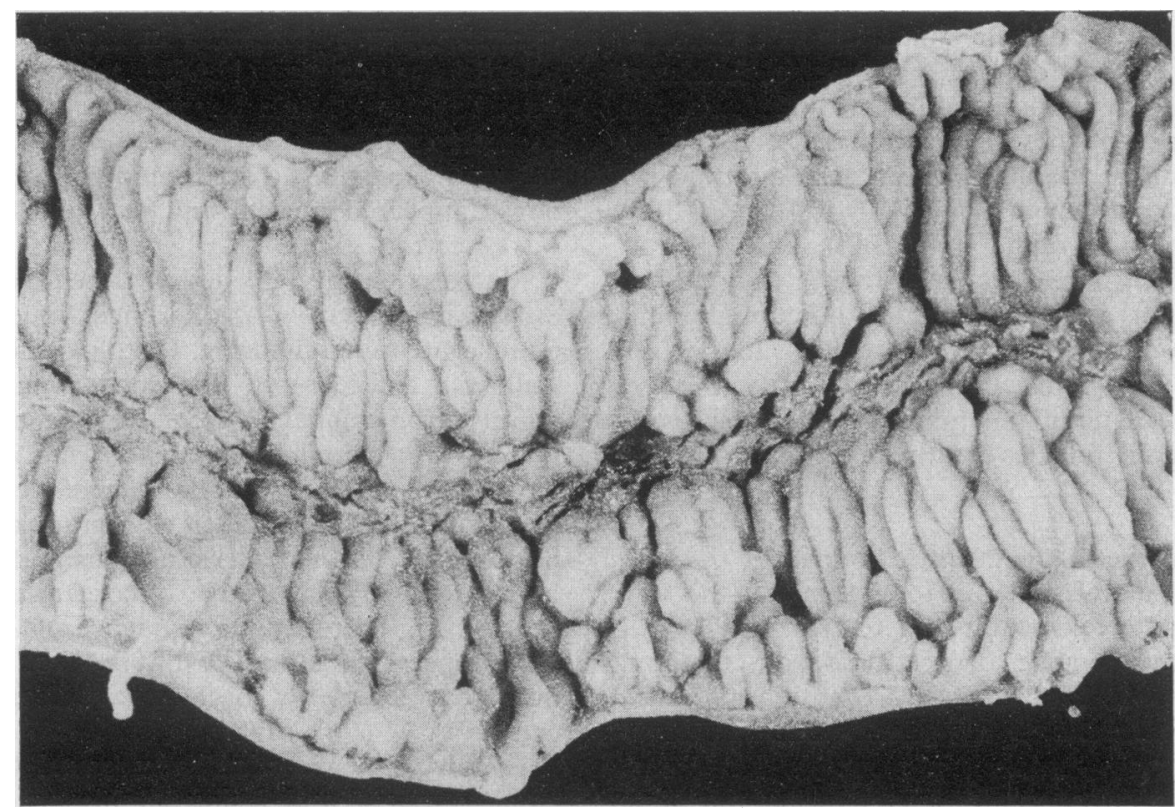

Fig. 3. Macroscopic appearance of resected jejunum. The mucosa is thickened and there are nodular areas with fissuring present.

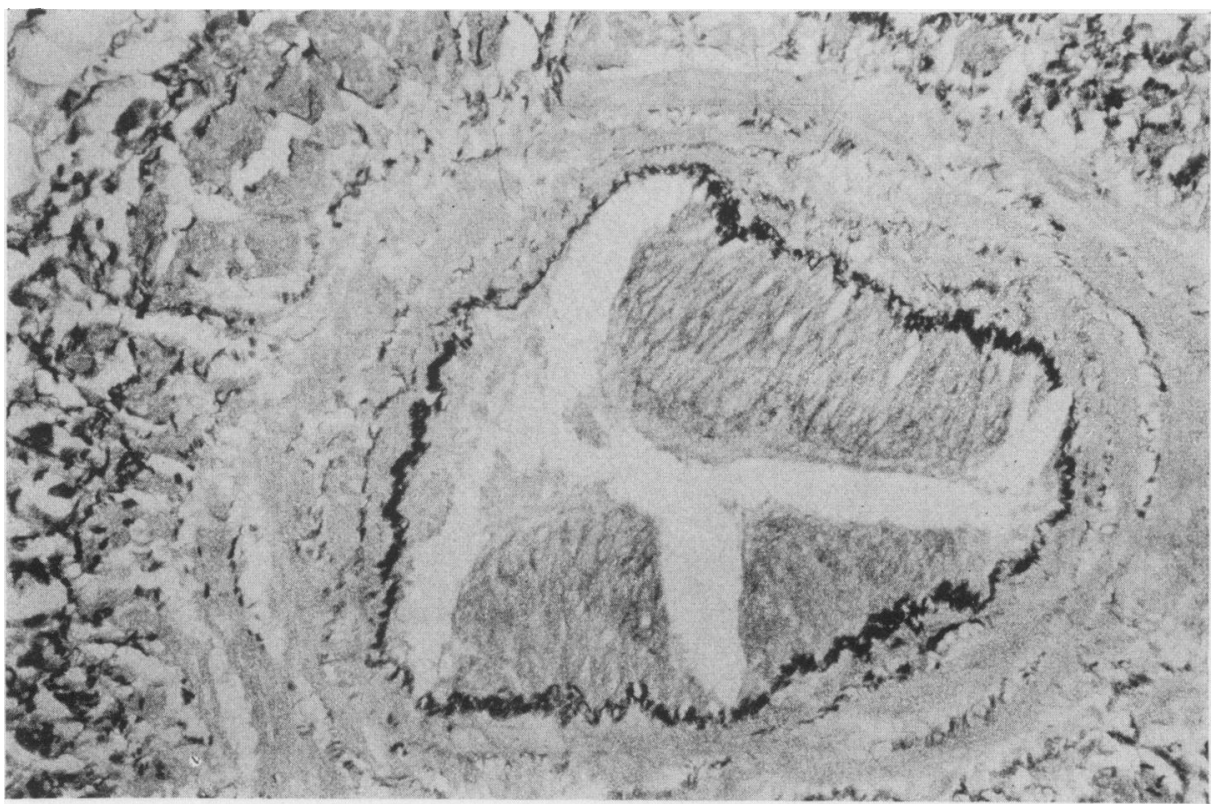

FIG. 4. Partially occluded mesenteric vein, Elastin Van Gieson $\times 120$. 


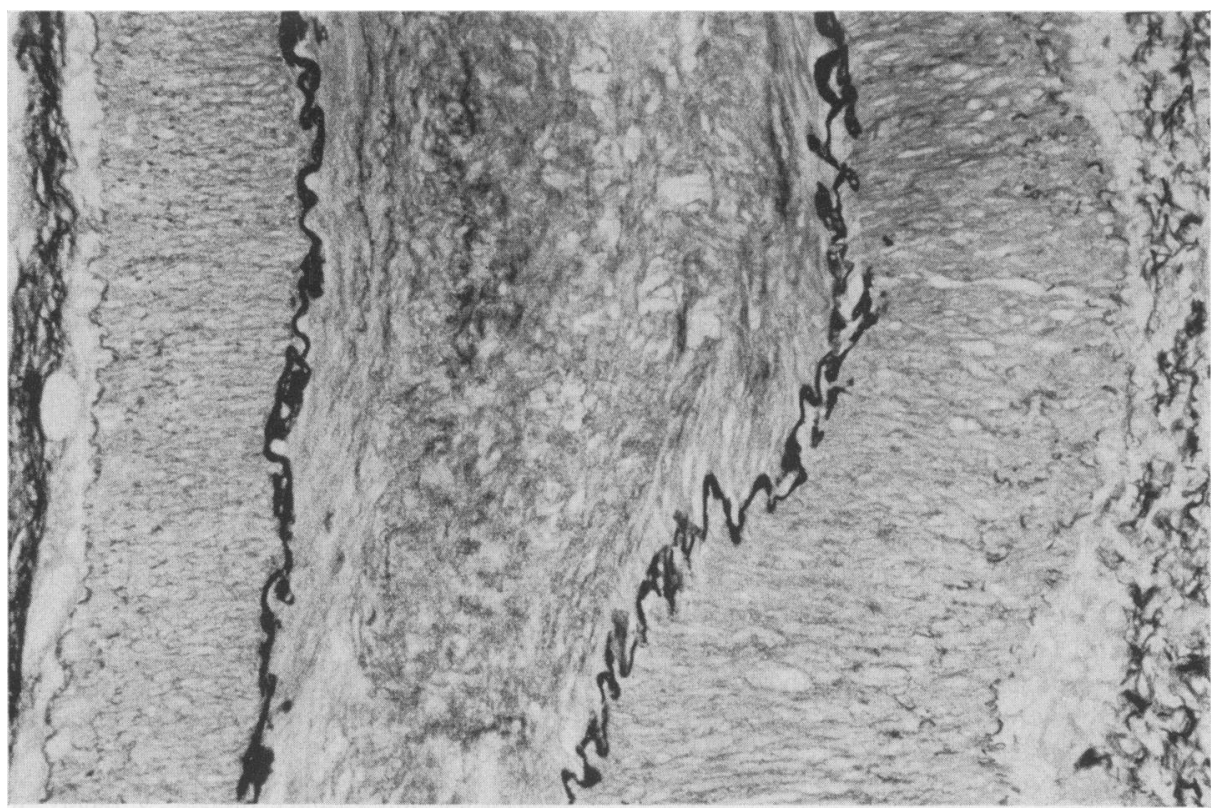

FIG. 5. Longitudinal section of mesenteric artery showing occlusion, Elastin Van Gieson $\times 120$.

\section{Discussion}

The main features of interest in this case are, firstly, the way this patient illustrates many of the well documented features of acquired immunodeficiency; secondly, the nature of the jejunal lesion; and, finally, the unique finding of widespread vascular occlusion in the resected intestine.

Late onset or acquired immunodeficiency, first described by Sanford, Favour and Tribeman (1954), Prasad and Koza (1954), Grant and Wallace (1954) and Zinneman, Hall and Heller (1954), is a condition characterized by abnormally low immunoglobulin levels and recurrent infections in patients who are not especially prone to infection for several years after birth. The presentation in the present patient at the age of 40 is typical, as is the long delay in reaching a final diagnosis.

This case showed the characteristic pattern of reduction in all 3 classes of immunoglobulin. All these patients have reduced serum IgG concentrations, although Hermans, Diaz-Buxo and Stabo (1976) have pointed out that unusual susceptibility to infection usually occurs only with levels below $3.75 \mathrm{~g} / \mathrm{l}$.

This patient initially presented with diarrhoea, a gastrointestinal complication which occurs in more than $50 \%$ of these patients, many of whom also have associated giardiasis. Several causes for this diarrhoea have been suggested, including overgrowth of the normal enteric flora (Ament and
Rubin, 1972), salmonellosis and shigellosis (Waldman and Laster, 1964), and giardiasis (Ament and Rubin, 1972; Waldmann and Laster, 1964; Hermans, Huizenga and Hoffman, 1966; Hughes et al., 1971; Ament, Ochs and Davis, 1973), but clearly do not account for all instances. In the patient described it is possible that the mechanical obstruction caused by the inflamed thickened bowel contributed to his giardiasis.

The multiple small filling defects throughout the small intestine in the present patient are typical of those seen in nodular lymphoid hyperplasia, being smooth, uniform in size and a few millimetres in diameter. The histological appearance of large lymphoid follicles within the lamina propria with normal overlying mucosa is characteristic. Germinal centres are seen within these follicles, and plasma cells are absent or markedly reduced in number. Different degrees of lymphoid hyperplasia are seen in immunodeficiency (Pelkonen, Siurala and Vuopio, 1963; Collins and Ellis, 1965) with the most proliferative form-nodular lymphoid hyperplasiaoccurring in more than $25 \%$ of those patients with associated diarrhoea. It is possible that the lymphoid hyperplasia is due to 'piling up' of B lymphocytes whose further differentiation into mature plasma cells is blocked (Michaels et al., 1971).

The jejunal lesion in the present patient consisted of transmural, chronic inflammation and fissures with associated mesenteric lymphadenitis. Crohn's 
disease is a possible diagnosis, although there are several points against this. Whilst there was a diffuse transmural chronic inflammatory reaction, no granulomata were seen histologically. There were no skip lesions and, furthermore, vascular occlusions in both arteries and veins were very prominent in this lesion and such a finding is not a recognized feature of Crohn's disease. The cause of the widespread vascular occlusion is not known. The thromboses of mesenteric arteries and veins could of course be secondary to the underlying inflammatory disease, and not in fact a primary abnormality. However, it is also possible that the vascular occlusions were responsible in part for the bowel lesion.

There are several reports on intestinal disorders in association with immunodeficiency states. Crohn's disease has been reported in association with generalized hypogammaglobulinaemia by several authors (Eggert, Wilson and Good, 1969; Fillit et al., 1977; Söltoft, Peterson and Kruse, 1972). In addition, selective $\operatorname{IgA}$ deficiency has been reported in association with Crohn's disease (Hodgson and Jewell, 1977).

Non-granulomatous ulcerative jejuno-ileitis has also been reported in association with immunodeficiency by Saxon et al. (1977). His 2 cases show several similarities to the case here reported. Both Saxon's cases presented with chronic diarrhoea, weight loss and malaise, and were found to have hypogammaglobulinaemia and small-bowel villous atrophy which failed to respond to a gluten-free diet. He showed that in his cases, the B cells were responsible for the failure of Ig production.

With regard to the other features in this case the cholelithiasis and transient arthritis are of interest since it is well recognized that gall stones have a higher prevalence, both in acquired immunodeficiency (Hermans et al., 1976) and in Crohn's disease. Transient seronegative arthritis occurs in inflammatory bowel disease, and can occur in acquired immunodeficiency states, although this is more common in the congenital form of the disease.

\section{Acknowledgments}

I would like to thank Professor L. A. Turnberg for his help and encouragement and $\operatorname{Dr} \mathrm{A}$. W. Jones for pathological photographs.

\section{References}

Ament, M.E., Ochs, H.D. \& Davis, S.D. (1973) Structure and function of the gastrointestinal tract in primar immunodeficiency syndromes: a study of 39 patients Medicine. Baltimore, 52, 227.

Ament, M.E. \& Rubin, C.E. (1972) Relation of giardiasis to $0^{\circ}$ abnormal intestinal structure and function in gastro $\overrightarrow{\bar{F}}$ intestinal immuno-deficiency syndromes. Gastroenterolog, 62, 216.

Collins, J.R. \& Ellis, D.S. (1965) Agammaglobulinemia malabsorption and rheumatoid-like arthritis. America Journal of Medicine, 39, 476.

EgGert, R.C., WiLson, I.D. \& Good, R.A. (1969) Agamma@ globulinemia and regional enteritis. Annals of Internasu Medicine, 71, 581.

Fillit, H., Bernstein, L., Davidson, M., BRANDT, L. $\overrightarrow{0}$ Bezahler, G. \& Cohen, M. (1977) Primary acquiredhypogammaglobulinemia and regional enteritis. Archive $\overrightarrow{\tilde{\omega}}$ of Internal Medicine, 137, 1252.

Grant, G.H. \& Wallace, W.D (1954) Agammaglobulin aemia. Lancet, ii, 671.

Hermans, P.E., Diaz-Buxo, J.A. \& Stobo, J.D. (1976 Idiopathic late-onset immunoglobulin deficiency: clinicaß observations in 50 patients. American Journal of Medicine's 61, 221.

Hermans, P.E., Kiuzenga, K.A. \& Hoffman II, H.N. (1966 Dysgammaglobulinemia associated with nodular lymphoid hyperplasia of the small intestine. American Journal of Medicine, 40, 78.

Hodgson, H.J.F. \& Jewell, D.P. (1977) Selective IgAdeficiency and Crohn's disease: a report of two cases Gut, 18, 644.

Hughes, W.S., Cerda, J.J., Holtzapple, P. \& Brooks, F.P六 (1971) Primary hypogammaglobulinemia and malabsorp tion. Annals of Internal Medicine, 74, 903.

Michaels, D.L., Go, S., Humbert, J.R., Dubois, R요. Stewart, J.M. \& Ellis, E.F. (1971) Intestinal nodu lymphoid hyperplasia, hypogammaglobulinemia hematological abnormalities in a child with a ring 780 chromosome. Journal of Pediatrics, 79, 80.

Pelkonen, R., Siurala, M. \& Vuopio, P. (1963) Inheritec agammaglobulinemia with malabsorption and marked alterations in the gastrointestinal mucosa. Acta medic (t) scandinavica, 173, 549.

Prasad, A.S. \& KozA, D.W. (1954) Agammaglobulinemia Annals of Internal Medicine, 41, 629.

SANFORd, J.P., Favour, C.B. \& Tribeman, M.S. (1954) Absence of serum gamma globulins in an adult. Ner穷 England Journal of Medicine, 250, 1027.

Saxon, A., Stevens, R.H., Ashman, R.F. \& Parker, N.H흐 (1977) Dual immune defects in non-granulomatous ulcerative jejuno-ileitis with hypogammaglobulinemia.: Clinical Immunology and Immunopathology, 8, 272.

Söltoft, J., Peterson, L. \& Kruse, P. (1972) Immunoglobulin deficiency and regional enteritis. Scandinavians Journal of Gastroenterology, 7, 233.

WALdmanN, T.A. \& LASTER, L. (1964) Abnormalities of albumin metabolism in patients with hypogammaglobulin $\rightarrow$ emia. Journal of Clinical Investigation, 43, 1025.

Zinneman, H.H., Hall, W.H. \& Heller, B.I. (1954) Acquired agammaglobulinemia: a report of three cases $N$ Journal of the American Medical Association, 156, 1390. 